\title{
Cellulose acetate electrospun nanofibrous membrane: fabrication, characterization, drug loading and antibacterial properties
}

\author{
NAZNIN SULTANA ${ }^{1,2, *}$ and ANISAH ZAINAL ${ }^{1}$ \\ ${ }^{1}$ Faculty of Bioscience and Medical Engineering, Universiti Teknologi Malaysia, 81300 Johor, Malaysia \\ ${ }^{2}$ Advanced Membrane Technology Research Center, Universiti Teknologi Malaysia, 81300 Johor, Malaysia
}

MS received 15 September 2015; accepted 29 October 2015

\begin{abstract}
Cellulose-based materials are one of the most commonly used materials for biomedical applications, which normally applied as carriers for pharmaceuticals and drug-releasing scaffolds. In this study, cellulose acetate (CA) was used to fabricate the nanofibrous membrane using the electrospinning technique. CA solutions at different concentrations were prepared by dissolving the polymer in a mixture of acetic acid/acetone solvents with the ratio of $3: 1$. The field emission scanning electron microscope results showed that electrospinning of $10 \%$ (w/v) CA produced nanofibres with many beads. When the CA concentration was increased to $14 \%(\mathrm{w} / \mathrm{v})$, bead-free nanofibres were produced. The contact angle measurement results confirmed the hydrophilic properties of nanofibres. In order to prevent common bacterial infections, a model drug, Tetracycline · HCL was incorporated into the CA nanofibres. The drug-loaded CA nanofibres showed antibacterial activity against Gram-positive and Gram-negative bacteria. CA nanofibres had high water uptake properties. The CA nanofibrous membrane was non-toxic to human skin fibroblast cells. Thus the CA nanofibres with $14 \%(\mathrm{w} / \mathrm{v})$ concentration exerted suitable properties for wound healing application.
\end{abstract}

Keywords. Electrospinning; scaffold; cellulose acetate; characterization; drug loading; antibacterial.

\section{Introduction}

In recent times, research on developing biodegradable, biofunctional and biocompatible nanosized materials has gained much interest. Nanometre length is the scale of proteins and other biological macromolecules, functional interactions between these molecules inside and outside of cells, and viruses. Hence, researchers are trying to fabricate nanosized biomaterials scaffolds which can mimic the living matter for various purposes including tissue engineering and wound dressing applications [1]. There are many techniques in fabricating biomaterial scaffolds. Among various techniques of scaffold fabrication, electrospinning technique is one of the most widely used techniques to fabricate highly porous fibrous scaffolds in micro- and nanoscale [2-4]. It is a nonmechanical processing strategy which is versatile and inexpensive [5]. Electrospun fibres produced can mimic cellular microenvironment and favourable for cell attachment, proliferation and differentiation. Studies have shown that fibrous scaffolds have more advantages over non-fibrous scaffolds [6]. The electrospinning technique uses high voltage electric field to create a charged jet of polymer solution and deposited on a grounded collector after travelling in the air [7]. When high voltage is applied to the polymer solution, electrostatic force will be formed and balances with surface tension forming a cone at the tip of the needle, called Taylor cone. With the increase in electric field, polymer solution forms fibre jet

*Author for correspondence (naznin@biomedical.utm.my) when electrostatic force is larger than surface tension. During the ejection and elongation of the fibre jet, solvent will be evaporated leaving only fibres which is attracted to the grounded collector $[7,8]$.

Various natural and synthetic polymers or a mixture of proteins, nucleic acids and polysaccharides have been used for electrospinning. Throughout the years, polymers have been electrospun successfully from several natural and synthetic polymers and characterized according to their applications [9]. Some of the polymers are cellulose, collagen, chitosan, chitin, polystyrene, polyvinyl pyrrolidone (PVP), polyvinyl alcohol (PVA), polylactic acid (PLA), polyester urethane (PEU) and polycaprolactone (PCL) [9-15]. Cellulose acetate (CA) fibres have in recent times gained attention in the field of biomedical application. Previous studies on cellulose acetate fibres underlining its application for ultrafiltration and drug-discharging scaffolds have been widely reported [16].

This study explored the ability to electrospin CA using various solvent systems and different polymer concentrations as a potential for wound dressing application. Drug loading ability and antibacterial activity of drug-loaded membrane were also investigated. The surface morphology of the fibres obtained was then analysed using a field emission scanning electron microscopy (FESEM), and the wettability properties of the fibres was investigated using the contact angle measurement. The water uptake properties and the antibacterial properties were also investigated. MTT assay was carried out for determining the cytocompability of the fibres. 


\section{Materials and methods}

\subsection{Materials}

Materials used were CA (white powder; average molecular weight of 50,000), glacial acetic acid, acetone, dichloromethane (DCM) and distilled water. CA, glacial acetic acid, acetone and DCM were purchased from Sigma Aldrich Co., USA.

\subsection{Methods}

2.2a Preparation of polymer solutions: CA was dissolved in three types of solvent systems in order to determine the suitable solvent system to be used. The solvent systems used were acetic acid/water $(3: 1)$, mixture of acetone/DCM $(1: 1)$ and acetic acid/acetone $(3: 1)$.

The preliminary concentration of CA used for determining the suitable solvent systems was $10 \% \mathrm{w} / \mathrm{v}$. The CA polymers in different solvents were stirred magnetically at room temperature until a clear solution was obtained. This CA polymer solution was electrospun and the fibres collected was observed using SEM. Once the suitable solvent system had been determined, the polymer solutions with different concentrations of CA $(10,12,13$ and $14 \%(w / v))$ were used to fabricate the fibres.

2.2b Electrospinning: Using a electrospinning equipment (NaBond Nanofibre Electrospinning Unit, China), the CA polymer solution was electrospun. The CA solution was taken in a syringe with blunt-end-needle attached to it. It was given a constant feeding rate at $2.5 \mathrm{ml} \mathrm{h}^{-1}$ using a syringe pump. The distance of the needle tip to collector was kept constant at $10 \mathrm{~cm}$ and 10 to $15 \mathrm{kV}$ of voltage was applied. As the grounded collector, aluminium foil was used.

2.2c Characterization of CA nanofibrous membrane: morphology: The physical properties and the morphology of the fibres obtained were examined using SEM (Hitachi TM 3000, Japan) and FESEM (Hitachi SU8020, Japan). The samples were sputter-coated before observation. The fibres were cut into square section and mounted on aluminium stub and observed at accelerating voltage of $15 \mathrm{kV}$. Using a Phenom Benchtop SEM \& EDS ProX, the fibres diameter was measured. A total number of 300 fibres were measured and a histogram was generated to represent the data.

Wettability: Contact angle system (VCA Optima AST Product Inc., USA) was used to investigate the wettability properties of the fibres. Briefly, $2 \mu$ l of distilled water was dropped on the fibres surface and was allowed to adhere on the surface. The time taken for the drop of the distilled water to absorb into the fibres and its angle over time were recorded.

Water uptake properties: The fibres were further characterized by determining its ability to absorb water. The fibres were cut into $1 \times 1 \mathrm{~cm}^{2}$ squares and their weights were measured. This is the dry weight of each sample. Then, each sample was immersed in a closed bottle containing $50 \mathrm{ml}$ of distilled water for 1,3 and $5 \mathrm{~min}$. After the immersion period, the samples were weighed to record its wet weight. The water uptake of the samples was calculated as follows:

$$
\text { Water uptake }(\%)=\frac{w_{\mathrm{w}}-w_{\mathrm{d}}}{w_{\mathrm{d}}} \times 100,
$$

where $w_{\mathrm{w}}$ is the sample's wet weight and $w_{\mathrm{d}}$ the sample's dry weight.

2.2d Drug loading and antibacterial test: A model drug, Tetracycline hydrochloride (Calbiochem, USA) was loaded to the CA membrane. About 2\% (w/v) of Tetracycline hydrochloride was dissolved in the prepared polymer solution of cellulose acetate in mixture of acetic acid/acetone $(3: 1)$. The solution was magnetically stirred until all tetracycline were dissolved. Then electrospinning was conducted as discussed in section $2.2 \mathrm{~b}$. The feeding rate was kept constant at $2.5 \mathrm{ml} \mathrm{h}^{-1}$ and the collector distance was set at $10 \mathrm{~cm}$. The voltage applied was kept at $20 \mathrm{kV}$.

Zone inhibition method was used to investigate the antibacterial activity of the fibres. Circular discs with the diameter of $6 \mathrm{~mm}$ were punched from the fibres. Microorganisms Escherichia coli (E. coli) and Bacillus cereus (B. cereus) were used as the model microorganism. Next, nut rient agar plates were inoculated with $1 \mathrm{ml}$ of bacterial suspension containing $10^{8} \mathrm{cfu} \mathrm{ml}^{-1}$ for each type of bacteria using the spread plate method. The fibres were then gently placed on the plate and were incubated at $37^{\circ} \mathrm{C}$ for $24 \mathrm{~h}$. The zone of inhibition was determined by measuring the clear area formed around each samples.

2.2e MTT assay: Human skin fibroblast (HSF) cells were used to determine the cell viability of cellulose acetate membrane. HSF cells were cultured in the DMEM supplemented with $10 \%$ FBS and $1 \%$ pen-strip. The cells were incubated in a humidified atmosphere containing $5 \% \mathrm{CO}_{2}$ with temperature of $37^{\circ} \mathrm{C}$. The fibres were cut into circular shape that fit into the 96-well plates. Three 96-well plates were prepared to conduct this study. The samples were placed in the plates and then sterilized by washing it three times with PBS and were UV for $2 \mathrm{~h}$ and left to dry for $24 \mathrm{~h}$. The HSF cells that were previously cultured were then seeded onto the samples with $1 \times 10^{4}$ cells per well. In total $200 \mu$ l of growth medium were added to each well. Cells were incubated for $48 \mathrm{~h}$ in a humidified atmosphere containing $5 \% \mathrm{CO}_{2}$ with temperature of $37^{\circ} \mathrm{C}$.

Once the incubation time limit reached, the medium was removed and washed with PBS to remove the residues. Next, the samples were fed with $200 \mu$ of fresh medium and 50 $\mu l$ of MTT solution. This step was done in a dark environment. The plates then were wrapped with aluminium foil and incubated for $4 \mathrm{~h}$ in a humidified atmosphere containing $5 \% \mathrm{CO}_{2}$ with temperature of $37^{\circ} \mathrm{C}$. Subsequently, $200 \mu \mathrm{l}$ of 
DMSO was added to dissolve the formazan crystals formed. The medium with MTT were removed prior to adding the DMSO. Lastly, the absorbance was measured at $570 \mathrm{~nm}$ by using a Glomax Multi Detection System microplate reader. The result was expressed as mean \pm standard deviation. The value of $t$-test was determined from the graph expression. $P$-value less than $0.05(P<0.05)$ was considered as significant.

\section{Results and discussion}

\subsection{Solvent system}

In order to determine the suitable solvent system, three types of solvent systems were used. The concentration of CA used was $10 \%(\mathrm{w} / \mathrm{v})$ and the dual-solvent systems used were acetic acid/water (3:1), acetone/DCM (1:1) and
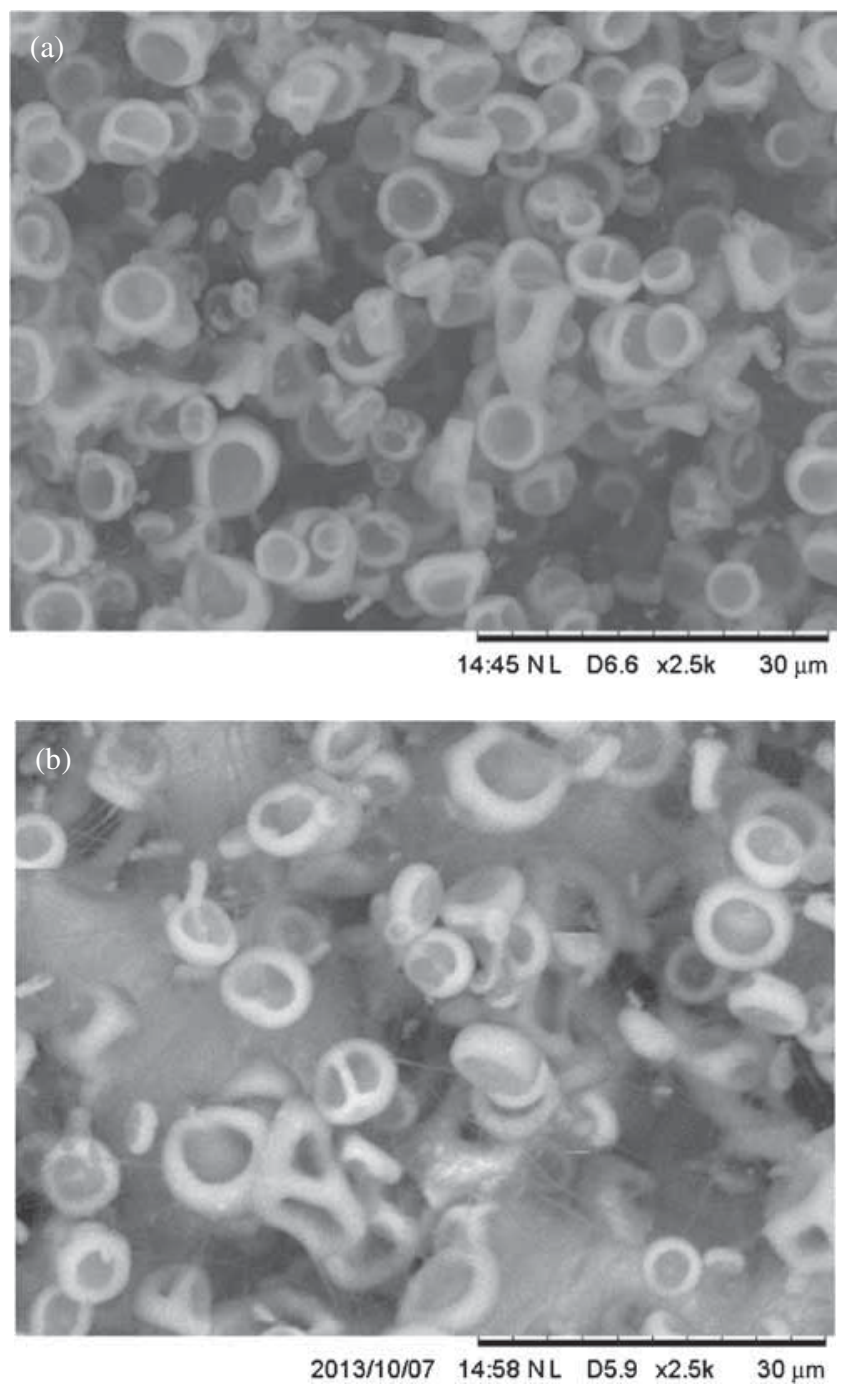

Figure 1. SEM images of polymer droplets of $10 \%(\mathrm{w} / \mathrm{v}) \mathrm{CA}$ obtained from (a) mixture of acetic acid/water and (b) mixture of acetic acid/acetone. acetic acid/acetone $(3: 1)$. Electrospinning of CA in the acetic acid/water generated a non-smooth jet whipping of the solution. Electrospinning of $\mathrm{CA}$ in the mixture of acetone/DCM (1: 1) was unsuccessful as no jet was generated and clogging of polymer droplets in the needle occurred. Figure 1 shows the SEM images of polymer droplets of $10 \%$ (w/v) CA solution obtained from mixture of acetic acid/water and mixture of acetic acid/acetone indicating unsuccessful production of fibres.

Although it was reported $[17,18]$ that CA can be electrospun in acetone/DCM and acetic acid/water, in this study the electrospinning of $\mathrm{CA}$ in mixture of acetic acid/water did not produce any fibres due to sensitivity of CA in contact with water and the $\mathrm{CA}$ in acetone/DCM solution was unable to produce fibres as DCM is a volatile liquid. Some other parameters including polymer concentration may affect the results. On the contrary, electrospinning of CA in acetic acid/acetone produced beaded-fibre and hence electrospinning of the CA was continued using the dual solvents acetic acid/acetone $(3: 1)$ as the solvent system.

\subsection{Morphology of fibres}

Electrospinning of CA was continued with different concentration of $\mathrm{CA}$ in order to determine the optimum concentration of CA. Different CA concentrations in the range of 10$14 \%(\mathrm{w} / \mathrm{v})$ in mixture of acetic acid/acetone was electrospun. Figure 2 shows the FESEM images of the electrospun CA with different concentration. Beads with few fibres were obtained from $10 \%$ CA while 12\% CA produced beaded fibres. Then the CA concentration was increased to 13 and $14 \%$. The $13 \%$ CA produced fibres with little beads while $14 \%$ CA produced densely packed ribbon-like fibres. As shown in figure 2, smooth CA fibres could be obtained when the concentration of $\mathrm{CA}$ is in suitable range.

The fibres diameter was in the range of 199.23-1056 nm (figure 3). This result showed that electrospinning of CA in the mixture of acetic acid/acetone can produced fine nanofibres.

\subsection{Surface wettability}

The surface wettability of the nanofibrous membrane was investigated using the water contact angle measurement. The fibres showed hydrophilic properties with time. The water first settled down onto the fibres for few seconds then rapidly absorbed into the fibres (figure $4 \mathrm{a}-\mathrm{d}$ ). From water contact angle measurement, it was observed that the water completely absorbed into the fibres within $40 \mathrm{~s}$ of exposure (figure 4e). This was supported by Liu et al [9] that concluded CA is hydrophilic.

\subsection{Water uptake properties}

The water uptake properties of the nanofibrous membranes were investigated. The fibres were immersed in distilled 

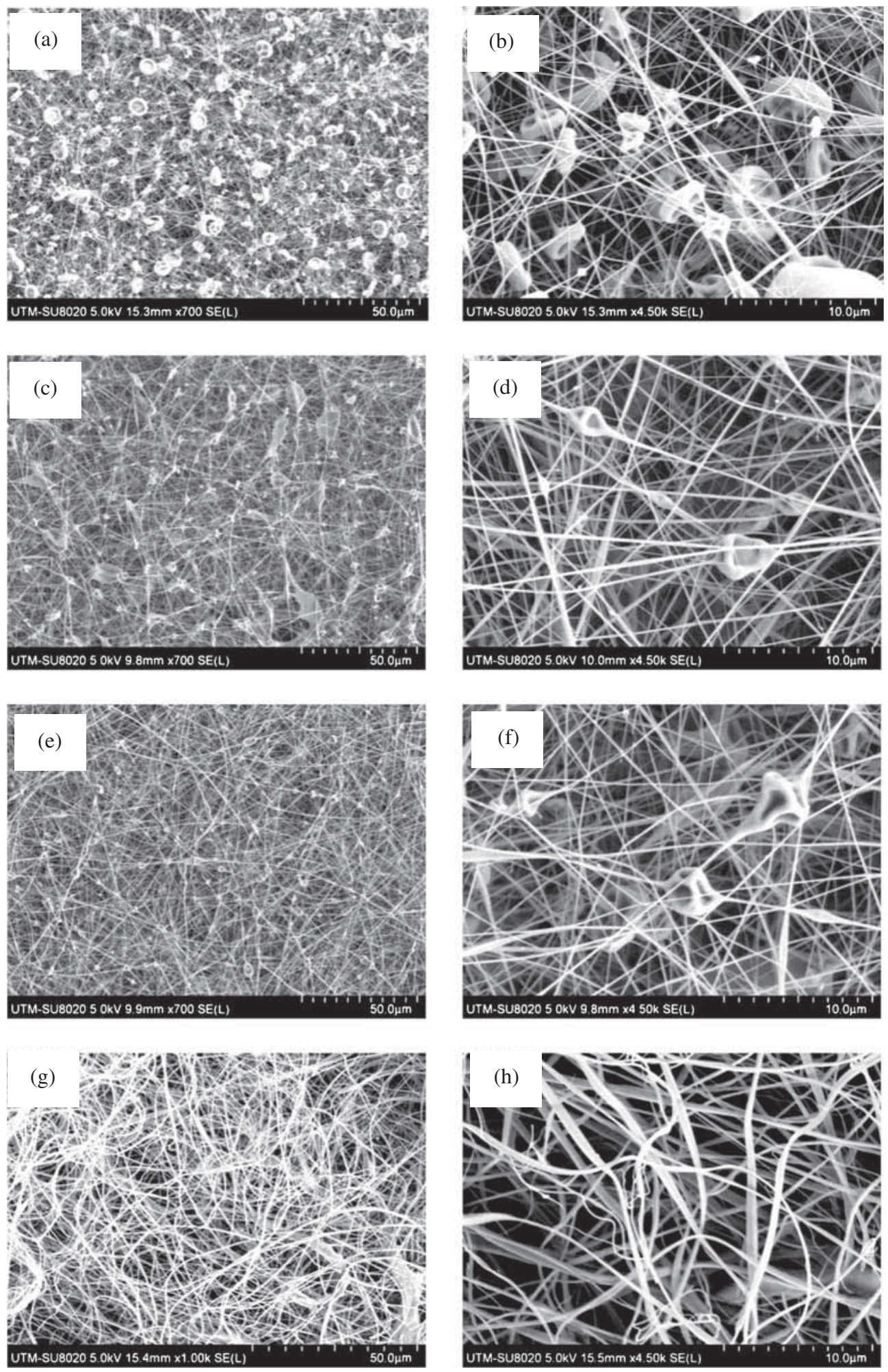

Figure 2. FESEM images of (a, b) $10 \%(\mathrm{w} / \mathrm{v}) \mathrm{CA},(\mathbf{c}, \mathbf{d}) 12 \%(\mathrm{w} / \mathrm{v}) \mathrm{CA},(\mathbf{e}, \mathbf{f}) 13 \%(\mathrm{w} / \mathrm{v}) \mathrm{CA}$ and $(\mathbf{g}, \mathbf{h}) 14 \%(\mathrm{w} / \mathrm{v}) \mathrm{CA}$ fabricated from mixture of solvents acetic acid/acetone $3: 1$ (a, $, \mathbf{c}, \mathbf{e}, \mathbf{g}$ : low magnification and $\mathbf{b}, \mathbf{d}, \mathbf{f}, \mathbf{h}$ : high magnification).

water for different interval of time. As the time increased the water uptake by the fibres increased (figure 5a). After about $5 \mathrm{~min}$, an equilibrium water uptake was observed which was confirmed by no further increase in weight. This showed that CA fibres can absorb and hold water and it is a good characteristic for application in wound 
dressing. The nanofibres retained their nanofibrous morphology and were not broken after the water uptake testing (figure 5b).

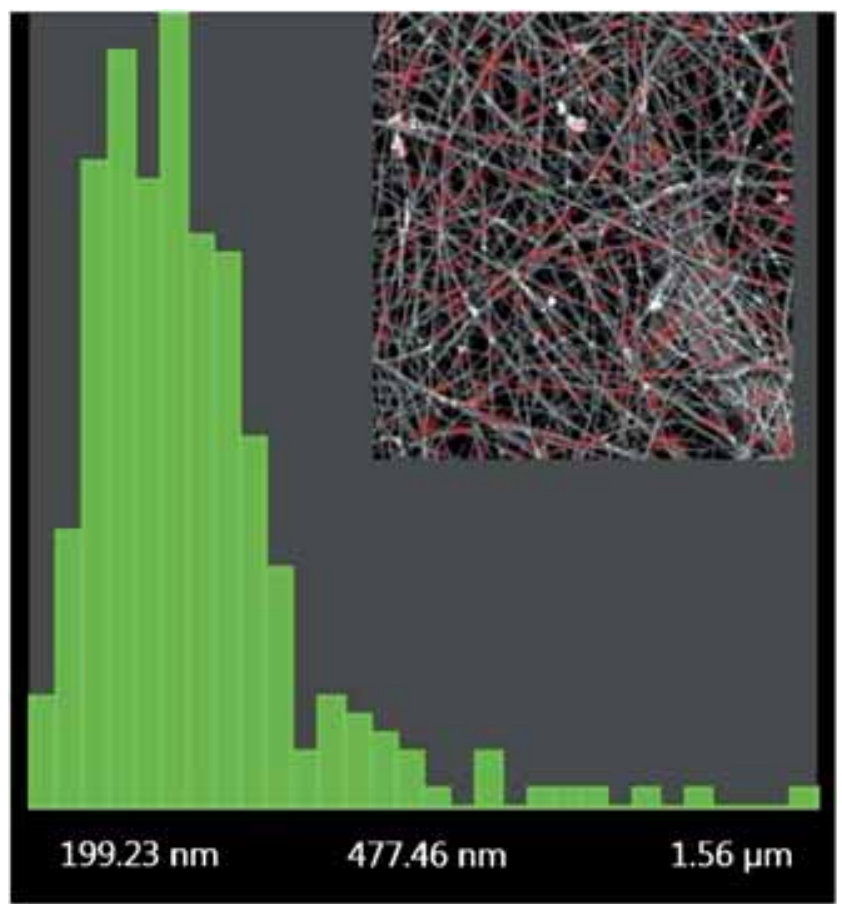

Figure 3. Distribution of the fibres diameter of membrane produced from $14 \% \mathrm{CA}$ in acetic acid/acetone $3: 1$.

\subsection{Antibacterial properties}

Tetracycline hydrochloride was incorporated into the CA nanofibrous membrane to test drug loading ability and antibacterial properties. Two types of bacteria were used; $B$. cereus and E. coli. Figure $6 \mathrm{a}$ and b shows the interaction of the bacteria when come in contact with the fibres. A significant clear area of inhibition was observed from plates containing drug incorporated membranes. The diameter of inhibition zones for B. cereus and $E$. coli were $4.1 \pm 0.1$ and $3.2 \pm 0.2 \mathrm{~cm}$ after $24 \mathrm{~h}$. On the other hand, no inhibition zone was observed in as-fabricated membrane containing no drug. Similar result was reported in another study about the effect of Tetracycline hydrochloride towards both bacteria mentioned [19]. Incorporation of the Tetracycline hydrocholride prevents bacteria from adhering to the membrane.

\subsection{MTT assay}

HSF cells were used to examine the fibres cytotoxicity. Figure 7 illustrates the MTT assay results for CA nanofibres and Tetracycline hydrochloride loaded nanofibres in comparison to control (tissue culture plastic, without membrane). It was observed that the cell viability was more than $90 \%$ of control after the nanofibres were seeded with HSF cells for 48 h. Rodriguez et al [14] also reported that cellulose fibres extract does not produce any cytotoxic effect on mammalian cells [20,21]. (a)

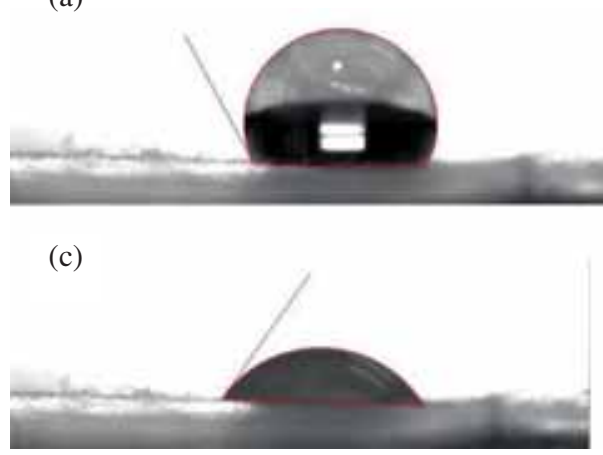

(b)

(e)

(d)

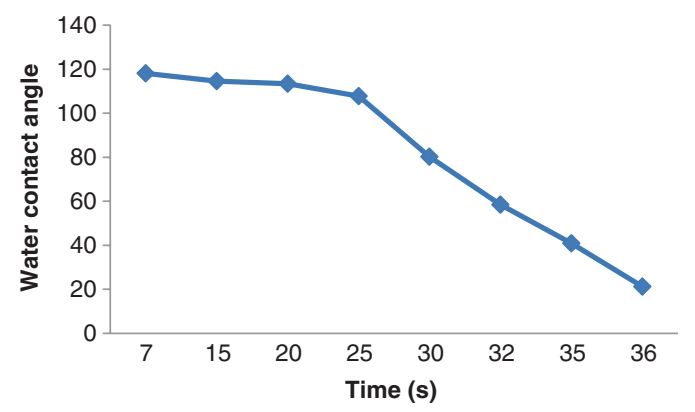

Figure 4. Water contact angle of CA nanofibres at (a) $7 \mathrm{~s}$, (b) $30 \mathrm{~s}$, (c) $32 \mathrm{~s}$, (d) $36 \mathrm{~s}$ and (e) decrease in water contact angle over time. 


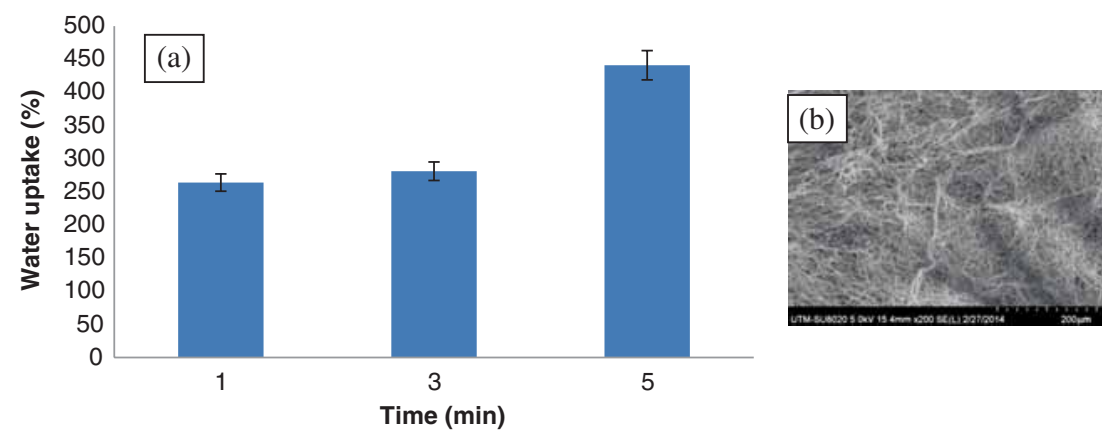

Figure 5. Water uptake properties of nanofibrous membrane fabricated from $14 \%$ (w/v) CA.
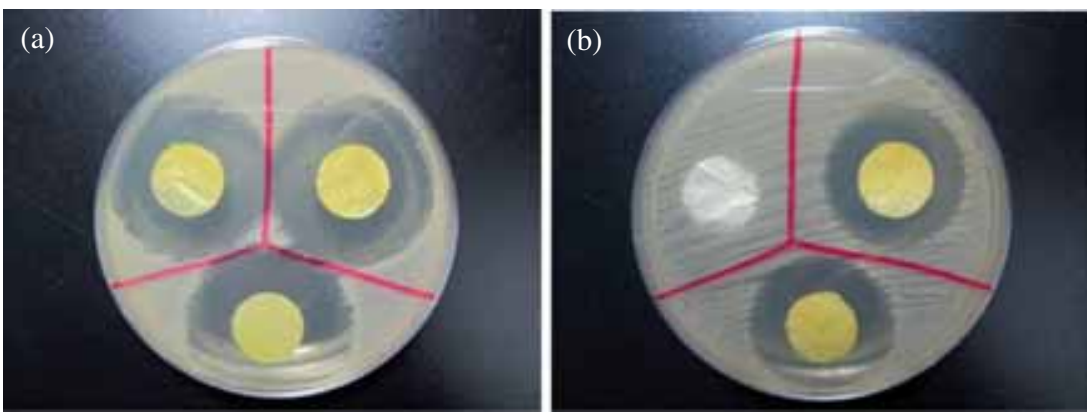

Figure 6. (a) Average diameter of inhibition of drug-loaded CA membrane $(4.1 \pm 0.1$ $\mathrm{cm})$ for B. cereus and (b) average diameter of inhibition $(3.2 \pm 0.2 \mathrm{~cm})$ in comparison to as-fabricated CA membrane for E. coli.

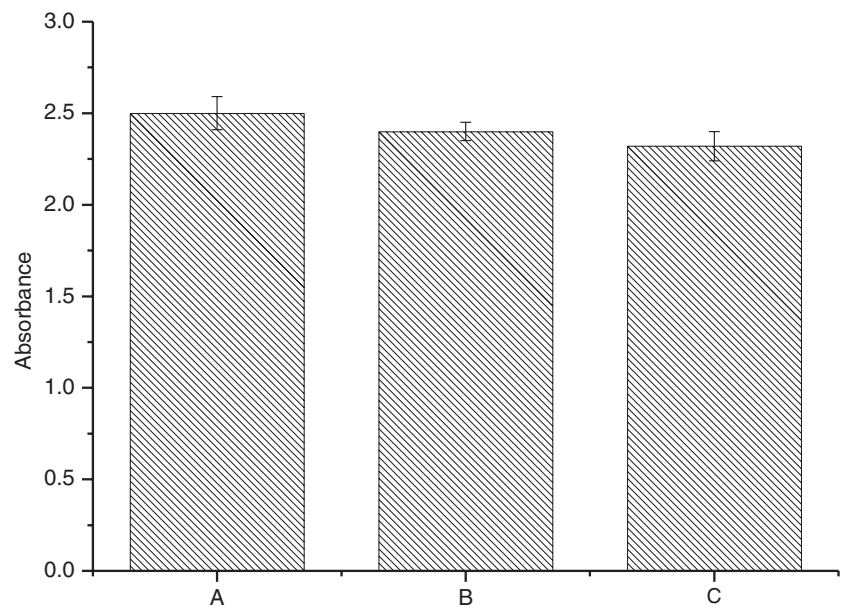

Figure 7. Cell cytotoxicity of control (A); CA nanofibres (B); tetracycline hydrochloride loaded $(\mathrm{C})$ nanofibres.

\section{Conclusions}

Different concentrations of CA $(10,12,13$ and 14\% (w/v)) were used to obtain nanofibres without beads. The concentration of $14 \% \mathrm{CA}$ produced beadless, densely packed ultrafine nanofibres. The characteristics of the fibres were investigated. The fibres were hydrophilic and exhibited high water uptake properties. Tetracycline hydrochloride-loaded nanofibrous membrane had good antibacterial properties. Besides, the MTT assay using HSF cells showed that the asfabricated and drug-loaded membranes were non-toxic to the cells.

\section{Conflict of interest}

The authors do not have any conflicts of interest.

\section{Acknowledgements}

We acknowledge MOHE, GUP Tier 1 grants (Vot: 12H24), AMTEC HiCOE grant (Vot: 4J191), FRGS (Vot: 4F507, MOHE), UTM and RMC for financial support. Lab facilities of FBME are also acknowledged.

\section{References}

[1] Miao J, Miyauchi M, Simmons T J, Dordick J S and Linhardt R J 2010 J. Nanosci. Nanotechnol. 105507

[2] Roozbahani F, Sultana N, Ismail A F and Nouparvar H 2013 J. Nanomater. 20131

[3] Hassan M I, Sun T and Sultana N 2014 J. Nanomater. 20147 
[4] Tong H-W and Wang M 2007 J. Nanosci. Nanotechnol. 73834

[5] Bhardwaj N and Kundu S C 2010 Biotechnol. Adv. 28325

[6] Bulasara V K, Uppaluri R and Purkait M K 2011 Mater. Manuf. Process. 26862

[7] Sill T J and von Recum H A 2008 Biomaterials 291989

[8] Prabhakaran M P, Venugopal J R, Chyan T T et al 2008 Tissue Eng. Part A 141787

[9] Liu X et al 2012 Biomed. Mater. Res. Part B 100B 1556

[10] Araujo J V et al 2008 Biomater. Sci. Polym. Ed. 191261

[11] Konwarh R et al 2013 Biotechnol. Adv. 31421

[12] Gouda M et al 2014 J. Ind. Textiles 43319
[13] Farack J et al 2011 Mater. Sci. Eng.: B 1761767

[14] Rodriguez K et al 2012 Cellulose 191583

[15] Sun T, Khan T H and Sultana N 2014 J. Nanomater. 20141 (Article ID 194680) doi: 10.1155/2014/194680

[16] Zhou W et al 2011 Open Mater. Sci. J. 551

[17] Celebioglu A and Uyar T 2011 Mater. Lett. 652291

[18] Han S O et al 2008 Mater. Lett. 62759

[19] Jin R M, Sultana N, Baba S, Hamdan S and Ismail A F 2015 J. Nanomater. 20151 (Article ID 357372) doi: $10.1155 / 2015 / 357372$

[20] Muller F A et al 2006 Biomaterials 273955

[21] Gouda M et al 2014 J. Ind. Textiles 43319 